\title{
Evaluation of Classical and Novel Ensemble Methods for Handwritten Word Recognition
}

\author{
Simon Günter and Horst Bunke \\ Department of Computer Science, University of Bern \\ Neubrückstrasse 10, CH-3012 Bern, Switzerland \\ sguenter, bunke@iam. unibe.ch
}

\begin{abstract}
Handwritten text recognition is one of the most difficult problems in the field of pattern recognition. Recently, a number of classifier creation and combination methods, known as ensemble methods, have been proposed in the field of machine learning. They have shown improved recognition performance over single classifiers. In this paper a number of ensemble methods for handwritten word recognition are described, experimentally evaluated, and compared to each other. Those methods include classical, general purpose ensemble methods as well as novel ensemble methods specifically developed by the authors for handwritten word recognition. The aim of the paper is to investigate the potential of ensemble methods for improving the performance of handwriting recognition systems. The base recognition systems used in this paper are hidden Markov model classifiers.
\end{abstract}

Keywords: ensemble methods, handwritten word recognition, hidden Markov model (HMM).

\section{Introduction}

The field of off-line handwriting recognition has been a topic of intensive research for many years. First only the recognition of isolated handwritten characters was investigated [26], but later whole words [25] were addressed. Most of the systems reported in the literature until today consider constrained recognition problems based on vocabularies from specific domains, e.g. the recognition of handwritten check amounts [13] or postal addresses [15]. Free handwriting recognition, without domain specific constraints and large vocabularies, was addressed only recently in a few papers $[16,22]$. The recognition rate of such systems is still low, and there is a need to improve it.

The combination of multiple classifiers was shown to be suitable for improving the recognition performance in difficult classification problems [18,27]. Also in handwriting recognition, classifier combination has been applied. Examples are given in $[20,28]$. Recently new ensemble creation methods have been proposed in the field of machine learning, which generate an ensemble of classifiers from a single classifier [3]. Given a single classifier, the base classifier, a set of classifiers can be generated by changing the training set [2], the input features [12], the 
input data by injecting randomness [4], or the parameters and the architecture of the classifier [23]. Examples of widely used methods that change the training set are Bagging [2] and AdaBoost [5]. Random subspace method [12] is a wellknown approach based on changing the input features. A summary of ensemble methods is provided in [3].

Although the popularity of multiple classifier systems in handwritten recognition has grown significantly, not much work on the use of ensemble methods for handwritten word recognition has been reported in the literature. This issue was recently addressed by the authors in a few papers [6, 7, 9-11]. In [6] ensemble methods using several prototypes were introduced. In [7] new boosting algorithms based on AdaBoost [5] were proposed. Ensemble methods using feature selection algorithms were introduced in [9] while a special combination scheme was described in [10]. Results of classical ensemble methods were reported in [11]. In the present paper we introduce a novel ensemble method and compare the most promising ensemble methods among each other, using a uniform framework for experimental evaluation. In contrast with earlier work reported by the authors, a more sophisticated base classifier is used. Also a new ensemble method is proposed (Subsection 3.2).

The rest of this paper is organized as follows. The base classifiers for handwritten word recognition used in the experiments are presented in Section 2. In Section 3, the ensemble methods evaluated in this paper are described. Then, in Section 4, experimental results of the ensemble methods with optimal parameter values are given. Finally, some conclusions are drawn in Section 5.

\section{Handwritten Word Recognizers}

The handwritten word recognizers used in this paper are similar to the one described in [22]. We assume that each handwritten word input to the recognizers has been normalized with respect to slant, skew, baseline location and height (for details of the normalization procedures see [22]). A sliding window of one pixel width is moved from left to right over the word and nine geometric features are extracted at each position of the window. Thus an input word is converted into a sequence of feature vectors in a 9-dimensional feature space. The geometric features used in the system include the fraction of black pixels in the window, the center of gravity, and the second order moment. These features characterize the window from the global point of view. The other features give additional information. They represent the position of the upper- and lowermost pixel, the contour direction at the position of the upper- and lowermost pixel, the number of black-to-white transitions in the window, and the fraction of black pixels between the upper- and lowermost black pixel.

For each uppercase and lowercase character, an HMM is build. For all HMMs the linear topology is used, i.e. there are only two transitions per state, one to itself and one to the next state. The character models are concatenated to word models. There is exactly one model for each word from the underlying dictionary. This approach makes it possible to share training data across different words. 
Two different versions of the classifier described in the previous two paragraphs, called $C_{1}$ and $C_{2}$ in the following, are employed in this paper. For classifier $C_{1}$ a fixed number of 14 states per HMM were used and the distributions of the features in each state of an HMM were modeled by single Gaussians. Four iterations of the Baum-Welch algorithm [24] were used for the training of the classifier. The number of states per HMM and the number of training iterations are optimal values (as determined by means of a validation set) under the assumption that the same number of states is needed for each individual character.

Classifier $C_{2}$ is a significantly refined version of classifier $C_{1}$. The number of states of the HMMs were optimized by the Quantile method [30] for each character individually. As a result, each individual character model has a different number of states. In addition the distributions of the features in each state of an HMM were modeled by Gaussian mixtures instead of single Gaussians. The training method of classifier $C_{2}$ was optimized on a validation set, using an optimization strategy described in [8].

\section{Ensemble Methods}

In this section the ensemble methods used in the experiments are described. The ensemble methods are divided in four categories: classical ensemble methods, partitions based ensemble method, novel boosting algorithms, and feature search ensemble method.

\subsection{Classical Ensemble Methods}

Two classical ensemble methods, AdaBoost and random subspace method were investigated in the experiments.

AdaBoost [5] modifies the original training set for the creation of the ensemble. Each classifier is trained on a different training set of the same size as the original training set. The main idea of AdaBoost is to concentrate the training on "difficult" patterns. The classical AdaBoost algorithm can only be used for two-class problems, but AdaBoost.M1 [5], a simple extension of AdaBoost, can cope with multi-class problems. Consequently, AdaBoost.M1 was applied in the experiments.

In the random subspace method [12] the individual classifiers use only a subset of all features for training and testing. The size of the subsets is fixed and the features are randomly chosen from the set of all features. In the experiments of this paper, 6 out of 9 features were selected, i.e. the fixed feature set size is 6 .

The classical ensemble Bagging [2] was also tested. As it produced inferior results, no description and no results of Bagging are given in this paper.

\subsection{Partitions Based Ensemble Method}

The basic algorithm of the partitions based ensemble methods can be described as follows. First, the whole training set is split into several partitions. Then each 
classifier is trained on all patterns of one of these partitions. This means that if the training set is split in $n$ partitions, $n$ different classifiers are obtained. There are two key parameters of this ensemble method. The first parameter is the algorithm actually applied to perform the partitioning, and the second is the number of partitions

For the experiments the partitioning was based on a clustering of the words according to their writing style. The whole training set was first clustered into groups of words with similar writing style where each group forms a partition. This was done by extracting some features of the handwritten text and applying the $k$-means clustering algorithm [14]. As we are using the IAM database [21], we always have complete pages of handwritten text, produced by the same writer, at our disposal (compare Section 4). Therefore the features were extracted from a whole page and all words of a page belong to the same cluster. The two features used for the clustering are the following:

- Words per component: The average number of words per connected component is calculated where small components are removed by a filtering procedure. A value of one of this feature corresponds to a complete cursive handwriting, i.e. the case where one connected component always represents exactly one word. By contrast, words consisting of isolated hand-printed characters have significantly lower values.

- Character width: The average width of the characters, in terms of pixels, is calculated. To this aim the lines of the pages are segmented in words using the procedure presented in [29]. The character width is then calculated as the sum of the lengths of the words of a page divided by the number of characters.

Both features were linearly normalized so that the mean of the features was 0 and the standard deviation was 1 .

The influence of the second parameter, the number $n$ of partitions, is as follows. The higher the number of $n$ is, the more similar are the writing stiles of the words of the same cluster. On the other hand, the average training set size decreases linearly with the number of clusters. As the performance of a system normally increases with the size of the training set, a rather high number of clusters will lead to classifiers with low performance. Usually, $n$ is a parameter the optimal value of which needs to be experimentally determined.

\subsection{Novel Boosting Algorithms}

As mentioned before, the original AdaBoost algorithm only works for two-class problems. AdaBoost.M1 [5] is a straightforward extension that basically regards the multi-class problem as a two-class problem with the two classes "correct" and "not correct". However, by doing this, we loose a great deal of information. In [7] three ensemble methods were introduced which are based on AdaBoost.M1, but which take all classes into account. The simple probabilistic boosting (SPB) defines the selection probability of a training pattern as a linear function of the 
likelihood of the misclassification of this pattern by the ensemble consisting of all already created classifiers. The effort based boosting (EBB) only trains with training elements for which the inclusion of instances of these elements leads, with a certain likelihood, to their correct recognition. Thus training elements which are always misclassified, even when including many of their instances in the training set, are not used for the training. The simple probabilistic boosting with effort (SPBE) is a combination of SPB and EBB. For more details of the three novel boosting algorithms we refer to [7].

\subsection{Feature Search Ensemble Method}

The feature search ensemble method described in this subsection was first introduced in [9]. The key idea of the method is not to select feature subsets for the individual classifiers of the ensemble randomly, as it is done in the random subspace methods [12], but to repeatedly apply an algorithm that selects a well performing features subset. In principle any known algorithm for feature selection can be used. In the ensemble method presented in this paper, a feature search starting with the empty set of features and a feature search starting with the full set of features were applied alternatively.

Similarly to most feature selection algorithms, the method applied in this paper tries to maximize the value of an objective function $f$. For a single classifier system this objective function is simply the classifier's performance on a validation set. However, in the present case we want to maximize the performance of the whole ensemble. Therefore the objective function $f$ measures the performance of the ensemble consisting of all classifiers which were already created and the classifier which is actually considered. The measurement of $f$ is performed on a separate validation set. Ideally $f$ also incorporates an estimation of the potential for improvement when adding more classifiers. In [9] two different objective functions were defined.

\section{Experiments}

For isolated character and digit recognition, a number of commonly used databases exist. However, for the task considered in this paper, there exists only one suitable database to the knowledge of the authors, holding a sufficiently large number of words produced by different writers [21]. Consequently this database was used in the experiments.

Two sets of experiments were done. The first set of experiments were conducted in order to evaluate the ensemble methods for a rather simple, straightforward base classifier. In these experiments the base classifiers $C_{1}$ was used. In the second set of experiments the highly optimized classifier $C_{2}$ was used as the base classifier. For the experiments of the second set the optimal number of classifiers was determined in a separate experiment.

To combine the individual classifiers of the ensembles the following combination schemes were applied: 
1. Voting scheme (voting): Only the top choice of each classifier is considered. The word class that is most often on the first rank is the output of the combined classifier. Ties are broken by means of the maximum rule (max) or the median rule $(m e d)$, which are only applied to the competing word classes.

2. Performance weighted voting (perf weight): Here we consider again the top class of each classifier. In contrast with regular voting, a weight is assigned to each classifier. The output of the combined classifier is the word class that received the largest sum of weights. The weight of a classifier is the performance of this classifier on the training set.

3. Weighted voting using optimized weights ( $g$ a weight): This scheme is similar to perf weight, but optimal weights are used which are calculated by a genetic algorithm based on the results of the classifiers achieved on the training set [19].

4. Special combination scheme for HMM-based recognizers (special): This combination was introduced in [10]. It integrates all HMMs of the different classifiers that correspond to the same character into a single HMM.

In the first set of experiments a data set of 10,927 words with a vocabulary of size 2,296 was used. That is, a classification problem with 2,296 different classes was considered. The total number of writers who contributed to this set is 81 . A training set containing 9,861 words and a test set containing 1,066 words were chosen in such a way that none of the writers of the test set were represented in the training set. Thus the experiments are writer independent. For the feature search ensemble method the training set was randomly split in a training set of 8,795 words and a validation set of 1,066 words. For this set of experiments the base classifier $C_{1}$, as described in Section 2, was used. The recognition rate of the base classifier $C_{1}$ was $66.23 \%$. The results of the ensemble methods are shown in Table 1. The name of the ensemble method is indicated in the column ensemble method. Please note that parameters of some ensemble methods were varied and only the results of the ensemble methods using the best parameter values, i.e. those which lead to the highest recognition rates on the test set, are shown. These parameter values are also given in the column ensemble method. (See [7,9] for details of these parameters). In column $c n$ the number of classifiers in the ensemble is given. The column combination scheme gives the scheme for which the highest recognition rate is obtained. The obtained recognition rate is finally shown in the last column.

Table 1 shows that the recognition rate of the base classifier was increased by $2.88 \%$ up to $5.81 \%$. The table furthermore shows that the ensemble methods of all categories are able to significantly improve the performance of the base classifier. We note that the classical ensemble methods were outperformed by all other ensemble methods specially developed by the authors for the domain of handwriting recognition.

In the second set of experiments a larger training set of 18,920 words and a larger test set of 3,264 words were used. The vocabulary of the experiments contains 3,997 words, i.e. a classification problem with 3,997 different classes is 
Table 1. Best results of the ensemble methods for base classifier $C_{1}$. The performance of $C_{1}$ is $66.23 \%$.

\begin{tabular}{|l|l|l|l|}
\hline ensemble method & cn & combination scheme & rec. rate. \\
\hline classical:AdaBoost & 14 & perf weight & $69.11 \%$ \\
\hline classical: random subspace method & 25 & ga weight & $69.35 \%$ \\
\hline partitions based & 5 & special & $70.83 \%$ \\
\hline boosting: EBB, $\alpha=1.5$ & 10 & ga weight & $69.82 \%$ \\
\hline feature search, $f_{2}$ & 10 & vote best med & $72.04 \%$ \\
\hline
\end{tabular}

Table 2. Best results of the ensemble methods for base classifier $C_{2}$. The performance of $C_{2}$ is $80.36 \%$.

\begin{tabular}{|l|l|l|l|}
\hline ensemble method & $\mathrm{cn}$ & combination scheme & rec. rate. \\
\hline classical: AdaBoost & 14 & vote max & $82.02 \%$ \\
\hline classical: random subspace method & 25 & vote med & $80.76 \%$ \\
\hline partitions based & 3 & special & $81.07 \%$ \\
\hline boosting: SPBE, $e_{3}$ & 14 & vote max & $82.69 \%$ \\
\hline feature search, $f_{2}$ & 13 & vote med & $82.69 \%$ \\
\hline
\end{tabular}

considered. The set of writers of the training set and the set of writers of the test set are disjoint, so the experiments are again writer independent. The total number of writers who contributed to this data set is 153 . For the feature search ensemble method the training set was randomly split three times in a training set of 17,920 words and a validation set of 1,000 words. The recognition rate of a system was calculated by averaging over the system's recognition rates obtained for each of the three splits. The base classifier $C_{2}$, as described in Section 2, is used. The recognition rate of the base classifier was $80.36 \%$.

The results of the second set of experiments are shown in Table 2 where the same notation as in Table 1 is used.

The recognition rate of the base classifier was increased by $0.4 \%$ up to 2.33 $\%$. This again shows that the ensemble methods of all categories are able to improve the performance of the base classifier. The random subspace method obtained only moderate improvements. The partitions based ensemble method did not perform as well as the classical method AdaBoost in this case. But both the boosting and the feature search ensemble methods were better than AdaBoost.

\section{Conclusions}

In this paper classical ensemble methods and novel ensemble methods specially developed for the domain of handwriting recognition were compared to each other. Two series of experiments were conducted. In the first series a rather simple and straightforward base classifier was used, while the base classifier of the second series was highly optimized. The results show that in both cases the performance of the base classifier can be significantly improved through the use 
of ensemble methods. The ensemble methods developed specifically for the task of handwriting recognition obtained either better results than the classical ones or produced much smaller ensembles. A possible future work is the evaluation of ensemble methods which use several base classifiers with different architectures. By applying an ensemble method on each base classifier and fusing the ensembles the performance may be increased even more. This was already confirmed in some earlier works for the case of two base classifiers. It may be promising to extend this approach to the case of more than two base classifiers.

\section{Acknowledgment}

This research was supported by the Swiss National Science Foundation (Nr. 20-52087.97). The authors thank Dr. Urs-Victor Marti for providing the handwritten word recognizer and Matthias Zimmermann for the segmentation of a part of the IAM database. Additional funding was provided by the Swiss National Science Foundation NCCR program "Interactive Multimodal Information Management (IM)2" in the Individual Project "Scene Analysis".

\section{References}

1. Proc of the 7th Int. Conf. on Document Analysis and Recognitio, Edinbourgh, Scotland, 2003.

2. Leo Breiman. Bagging predictors. Machine Learning, (2):123-140, 1996.

3. T. G. Dietterich. Ensemble methods in machine learning. In [17], pages 1-15.

4. T.G. Dietterich and E.B. Kong. Machine learning bias, statistical bias, and statistical variance of decision tree algorithms. Technical report, Departement of Computer Science, Oregon State University, 1995.

5. Yoav Freund and Robert E. Schapire. A decision-theoretic generalisation of online learning and an application to boosting. Journal of Computer and Systems Sciences, 55(1):119-139, 1997.

6. S. Günter and H. Bunke. Generating classifier ensembles from multiple prototypes and its application to handwriting recognition. In [18], pages 179-188.

7. S. Günter and H. Bunke. New boosting algorithms for classification problems with large number of classes applied to a handwritten word recognition task. In [27], pages $326-335$.

8. S. Günter and H. Bunke. Optimizing the number of states, training iterations and Gaussians in an HMM-based handwritten word recognizer. In [1], volume 1, pages $472-476$.

9. S. Günter and H. Bunke. Creation of classifier ensembles for handwritten word recognition using feature selection algorithms. In Proc. of the 8th International Conference on Frontiers in Handwriting Recognition, pages 183-188, 2002.

10. S. Günter and H. Bunke. A new combination scheme for HMM-based classifiers and its application to handwriting recognition. In 16th International Conference on Pattern Recognition, volume 2, pages 332-337, Quebec, Canada, 2002.

11. S. Günter and H. Bunke. Ensembles of classifiers for handwritten word recognition. International Journal of Document Analysis and Recognition, 5(4):224-232, 2003. 
12. T. K. Ho. The random subspace method for constructing decision forests. IEEE Transactions on Pattern Analysis and Machine Intelligence, 20(8):832-844, 1998.

13. S. Impedovo, P. Wang, and H. Bunke, editors. Automatic Bankcheck Processing. World Scientific Publ. Co, Singapore, 1997.

14. A. Jain, M. Murty, and P. Flynn. Data clustering: A survey. ACM Computing Survey, 31:264-323, 1999.

15. A. Kaltenmeier, T. Caesar, J.M. Gloger, and E. Mandler. Sophisticated topology of hidden Markov models for cursive script recognition. In Proc. of the 2nd Int. Conf. on Document Analysis and Recognition, Tsukuba Science City, Japan, pages 139-142, 1993.

16. G. Kim, V. Govindaraju, and S.N. Srihari. Architecture for handwritten text recognition systems. In S.-W. Lee, editor, Advances in Handwriting Recognition, pages 163-172. World Scientific Publ. Co., 1999.

17. J. Kittler and F. Roli, editors. First International Workshop on Multiple Classifier Systems, Cagliari, Italy, 2000. Springer.

18. J. Kittler and F. Roli, editors. Third International Workshop on Multiple Classifier Systems, Cagliari, Italy, 2002. Springer.

19. L. Lam, Y.-S. Huang, and C. Suen. Combination of multiple classifier decisions for optical character recognition. In H. Bunke and P. Wang, editors, Handbook of Character Recognition and Document Image Analysis, pages 79-101. World Scientific, 1997.

20. D. Lee and S. Srihari. Handprinted digit recognition: A comparison of algorithms. In Third International Workshop on Frontiers in Handwriting Recognition, pages 153-162, 1993.

21. U. Marti and H. Bunke. The IAM-database: An English sentence database for offline handwriting recognition. Int. Journal of Document Analysis and Recognition, 5:39-46, 2002.

22. U.-V. Marti and H. Bunke. Using a statistical language model to improve the performance of an HMM-based cursive handwriting recognition system. Int. Journal of Pattern Recognition and Art. Intelligence, 15:65-90, 2001.

23. D. Partridge and W. B. Yates. Engineering multiversion neural-net systems. Neural Computation, 8(4):869-893, 1996.

24. L. Rabiner. A tutorial on hidden Markov models and selected applications in speech recognition. Proceedings of the IEEE, 77(2):257-285, 1989.

25. J.-C. Simon. Off-line cursive word recognition. Special Issue of Proc. of the IEEE, 80(7):1150-1161, July 1992.

26. C.Y. Suen, C. Nadal, R. Legault, T.A. Mai, and L. Lam. Computer recognition of unconstrained handwritten numerals. Special Issue of Proc. of the IEEE, 80(7):1162-1180, 1992.

27. T. Windeatt and F. Roli, editors. 4th Int. Workshop on Multiple Classifier Systems, Guildford, United Kingdom, 2003. Springer.

28. L. Xu, A. Krzyzak, and C. Suen. Methods of combining multiple classifiers and their applications to handwriting recognition. IEEE Transactions on Systems, Man and Cybernetics, 22(3):418-435, 1992.

29. M. Zimmermann and H. Bunke. Automatic segmentation of the IAM off-line database for handwritten English text. In Proc. of the 16th Int. Conference on Pattern Recognition, volume 4, pages 35-39, Quebec, Canada, 2002.

30. M. Zimmermann and H. Bunke. Hidden markov model length optimization for handwriting recognition systems. In Proc. of the 8th International Workshop on Frontiers in Handwriting Recognition, pages 369-374, 2002. 